\title{
Effect of processing conditions on physicochemical properties of astaxanthin nanodispersions.
}

\begin{abstract}
A top-down approach based on an emulsification-evaporation technique was used to prepare nanodispersions of astaxanthin. Response-surface methodology was employed to investigate the effect of the main processing conditions, namely, the applied pressure (20-90 MPa), number of cycles $(0-4)$ and evaporation temperature $\left(16-66^{\circ} \mathrm{C}\right)$, on the average particle size, polydispersity index and astaxanthin concentration of the nanodispersions. Second-order polynomial regression models expressing the astaxanthin nanodispersion properties as functions of the main processing variables were significantly $(\mathrm{p}<0.05)$ fitted, with high coefficient-of-determination values $(\mathrm{R} 2>0.90)$. A multiple-optimisation procedure showed that the optimum conditions of pressure, number of cycles of homogenization and evaporation temperature, were $50 \mathrm{MPa}$, two cycles and $47^{\circ} \mathrm{C}$, respectively. A statistical assessment showed insignificant $(\mathrm{p}>0.05)$ differences between experimental and predicted values, thus verifying the adequacy of the final reduced models fitted for explaining the variation of emulsion properties, as a function of homogenization and evaporation conditions.
\end{abstract}

Keyword: Emulsification-evaporation; Nanodispersion; Astaxanthin; High-pressure homogenizer; Average particle size; Polydispersity index. 